\title{
Theme: Neonatology
}

Trends in neonatal mortality (1990-2017), with projections to 2030 (Lancet Glob Health. 2019; 7:e710-20)

Improving neonatal mortality rate (NMR) is an essential component of reducing under-5 mortality rate (U-5MR). To achieve the current target of Sustainable Development Goals (SDGs) of ending preventable deaths of neonates and under-5 children by 2030 , all countries should aim to reduce the NMR to 12 deaths/1000 livebirths. This systematic analysis estimated how different NMR targets could affect the actual burden of neonatal mortality from 2018 to 2030 using the present NMR data of 195 countries. Between 1990 and 2017, the global NMR decreased by $51 \%$ (36.6 deaths/1000 livebirths in 1990 to $18 \cdot 0$ deaths/1000 livebirths in 2017). Total neonatal deaths during the same period decreased from $5 \cdot 0$ to $2 \cdot 5$ million. West and Central Africa had the highest NMR in 2017 - at $30 \cdot 2$ deaths $/ 1000$ livebirths - followed by South Asia, at 26.9 deaths/1000 livebirths. Though all regions have reported reductions in NMR since 1990, South Asia, including India, reported a consistently and significantly higher ratio of NMR to U-5MR over the last 25 years. It has been projected that between 2018 and 2030, $27 \cdot 8$ million neonates will die if each country maintains its current rate of reduction in NMR. If each country achieves the SDG NMR target by effective implementation of continuum of care during pre-pregnancy, antenatal, intrapartum, delivery, postpartum, and postnatal periods for mothers and their newborns, neonatal deaths will reduce to $1 \cdot 2$ million in 2030 .

Increased early enteral zinc intake improves weight gain in hospitalized preterm infants (Acta Pediatr. May 01, 2019.doi.org/10.1111/apa.14828)

This cohort study showed that enteral zinc intake during neonatal intensive care unit (NICU) stay was associated with improved growth in 105 preterm infants. Multiple regression analysis showed that higher total enteral zinc intake was positively correlated with increase in weight and head circumference, after correcting post-menstrual age at birth.

Oxygen saturation alarm settings, and morbidity and mortality in preterm infants (JPediatr. 2019;209:17-22)

Even after five large randomized controlled trials, the choice of lower (85\%-89\%) vs. higher (91\%-95\%) target pulse oximetry oxygen saturation $\left(\mathrm{SpO}_{2}\right)$ range to reduce mortality and morbidity of preterm infants remains elusive. This retrospective cohort study evaluated the impact of policy changes (narrowing the $\mathrm{SpO}_{2}$ alarm limits) in very preterm infants. Records of 3809 infants in 10 hospitals with an $\mathrm{SpO} 2$ alarm policy change, and 3685 infants in 9 hospitals without a policy change, were reviewed. Mortality was lower in hospitals without a policy change (Adjusted Odds Ratio (aOR) 0.63; 95\% CI 0.50, 0.80) but did not differ between epochs in hospitals with changed policy. The odds of bronchopulmonary dysplasia were greater for hospitals with a policy change (aOR $1.65 ; 95 \%$ CI $1.36,2.00)$ but did not differ for hospitals without a policy change. Severe retinopathy of prematurity (ROP) and necrotizing enterocolitis did not differ between epochs for either group. Authors concluded that changing $\mathrm{SpO}_{2}$ alarm policies was not associated with reduced mortality or increased/severe ROP among very preterm infants.

Association between borderline low Apgar scores, and
neonatal mortality and morbidity in term infants
$(B M J .2019 ; 365: l 1656)$

This population-based cohort study examined the associations between 1, 5, and 10 minutes Apgar scores of 7, 8 and 9 versus 10 with neonatal mortality and morbidity in 1,551,436 normal term live singleton infants born between 1999 and 2016 with Apgar scores of $\geq 7$ at 1,5 , and 10 minutes. Compared to infants with an Apgar score of 10, neonatal mortality, infections, post-asphyxial complications, respiratory distress, and hypoglycemia were higher among infants with lower Apgar scores, especially at 5 and 10 minutes. The authors concluded that target Apgar score for all newborns should be 10 at each point of time as a small change in Apgar score from 5 minutes to 10 minutes, even within the normal range of 7 to 9 , was linked to higher neonatal morbidity risks.

Community-based antibiotic delivery for possible serious bacterial infections in neonates in low- and middle-income countries (Cochrane Database Syst Rev. 2019;4:CD007646)

This meta-analysis assessed the effects of community-based antibiotics for neonatal possible serious bacterial infection (PSBI) in low- and middle-income countries (LMICs), and whether such effects differ according to the antibiotic regimen. It was found that community-based antibiotic delivery for neonatal PSBI reduced neonatal mortality compared to hospital referral (RR $0.82,95 \%$ CI 0.68 to 0.99 ; 5 studies, $n=125,134$; lowquality evidence). It also showed a possible effect on reducing sepsis-specific neonatal mortality (RR $0.78,95 \%$ CI 0.60 to 1.00 ; 2 studies, $n=40,233$; low-quality evidence). Using a simplified antibiotic approach resulted in similar rates of neonatal mortality when compared to the standard 7-day regimen of injectable procaine benzyl penicillin and injectable gentamicin delivered in community-settings for neonatal PSBI (RR $0.81,95 \%$ CI 0.44 to $1.50 ; 3$ studies, $n=3476$; moderatequality evidence). Other approaches including 7-day regimen of oral amoxicillin and injectable gentamicin or 2-day regimen of injectable benzylpenicillin and injectable gentamicin or injectable gentamicin and oral amoxicillin, followed by oral amoxicillin for 5 days, also showed no difference in neonatal mortality.

SRIPARNA BASU drsriparnabasu@rediffmail.com 